\title{
Cost of Fault-Tolerance on Data Stream Processing
}

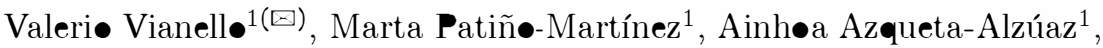 \\ and Ricard• Jimenez-Péris ${ }^{2}$ \\ 1 Universidad P•litécnica de Madrid, Madrid, Spain \\ $\{$ vvianello, mpatino, aazqueta\}@fi.upm.es \\ 2 LeanXcale, Madrid, Spain \\ rjimenez@leanxcale.com
}

\begin{abstract}
Data streaming engines precess data $\bullet$ the fly in cøntrast t•

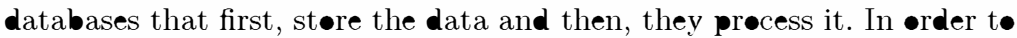
prøcess the increasing amøunt $\bullet$ data prøduced every day, data stream-

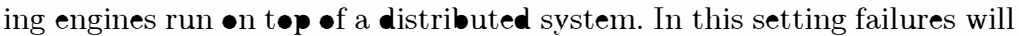
likely happen. Current distributed data streaming engines like Apache

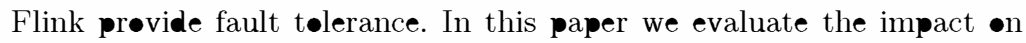
perførmance of fault tølerance mechanisms of Flink during regular •per-

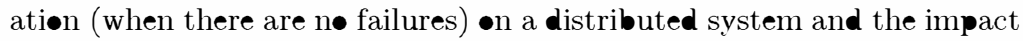
on performance when there are failures. We use the Intel HiBench for conducting the evaluation.
\end{abstract}

\section{Introduction}

Data streaming has become a popular data processing mødel in the last decade with the increase of the amøunt of data that is produced every second that must be processed on the fly. Typical examples of streaming applicatiøns include quick detection of price changes in the stock market, credit card fraud detection,

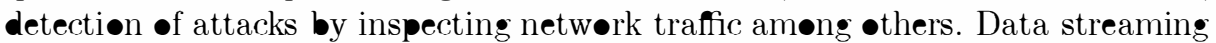

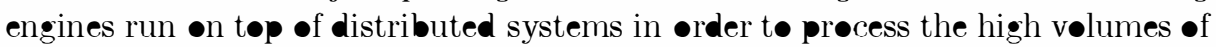

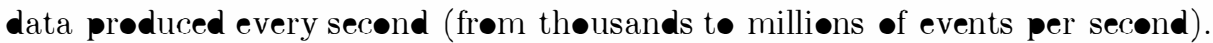
Distributed streaming engines like StreamCløud [8], Borealis [4] and Flink [1]

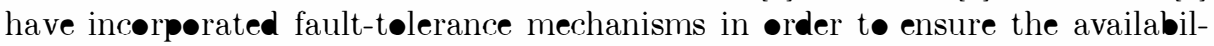

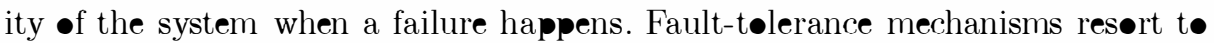
checkpointing the state of the data streaming application and the data streams in $\bullet$ rder t॰ be replayed when the system recevers after the failure, ensuring that all the data is processed. In this paper, we evaluate the performance everhead that the fault-tølerance mechanisms intrøduce during regular øperatiøn running the Intel HiBench benchmark [10] with Flink on top of a distributed system. We alse evaluate the time the system needs to resume regular processing and the impact on performance till the system returns t• regular •peration (the 
system precesses all the queued records during the failure). These performance

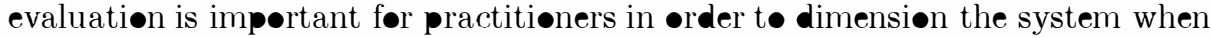

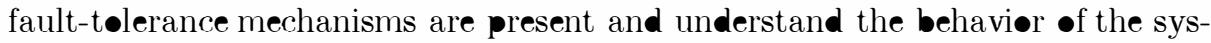
tem when it recevers.

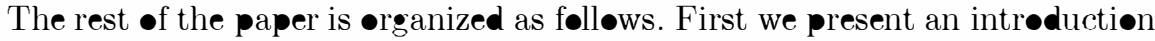
t• Flink (Sect.2) then, the fault-tølerance mechanisms •f Flink are described (Sect. 3). Sectiøn 4 presents the performance evaluation. Finally conclusiøns are presented in Sect. 5 .

\section{$2 \quad$ Flink Architecture}

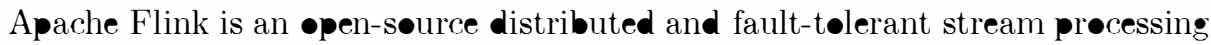

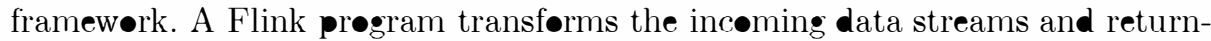
ing results thrøug sinks that can write them to different destinations. The

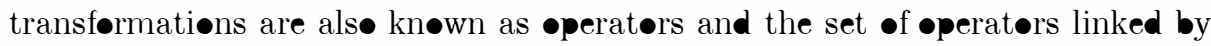

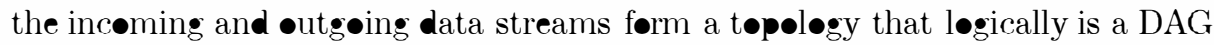
(directed acyclic graph).

Flink prøvides several built-in •peratørs which can be classified as

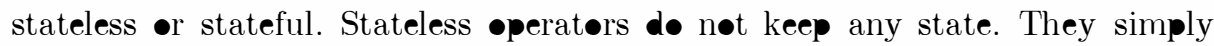

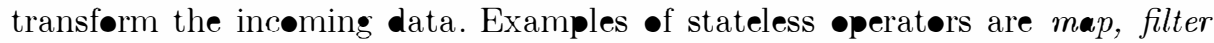

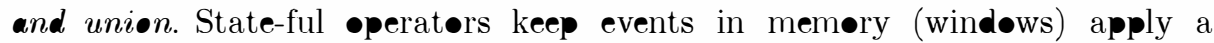
function and prøduce an øutput (time windows) or a number of records are received (record based windows). Examples of stateful operators are fold, aggregates, join.

At the core of the Flink architecture there are tw components that are

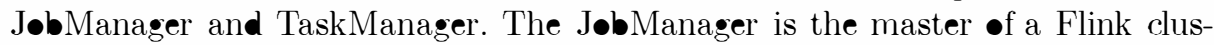

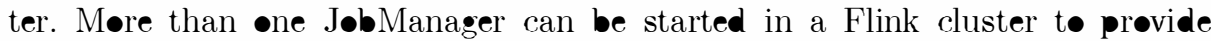

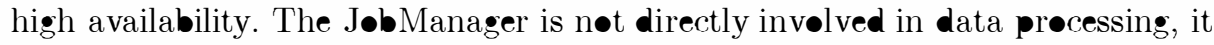
is in charge of coordinating the distributed execution. The TaskManager runs

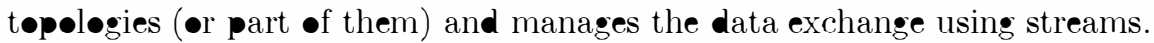

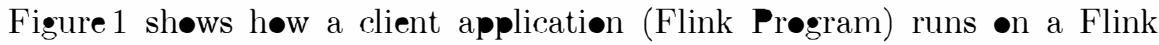

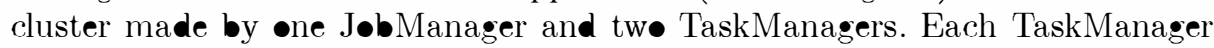

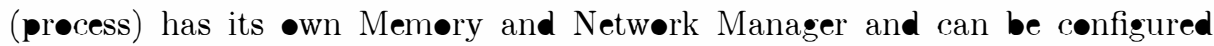

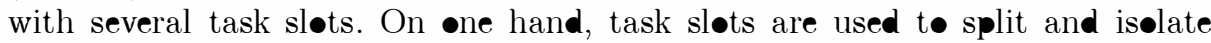
TaskManager dedicated memøry for different tøpoløgies. On the other hand,

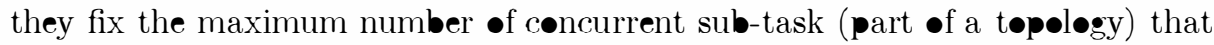
can be running on a given TaskManager. In Fig. 1, TaskManagers are configured with three task slots, it means that three sub-tasks from three different

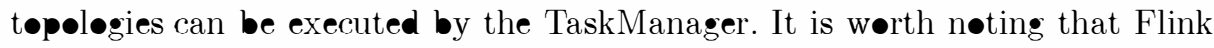

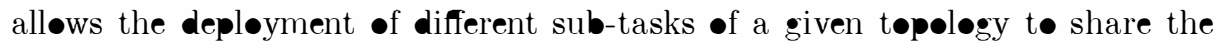

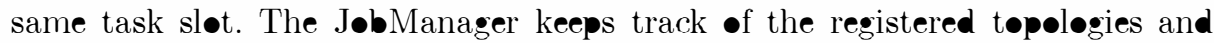
their corresponding dataflow graph. It alse schedules the tasks and decides on which TaskManager they are executed. On the client side, a Flink program is

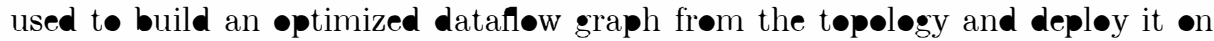
the Flink cluster sending it t• the JøbManager. 


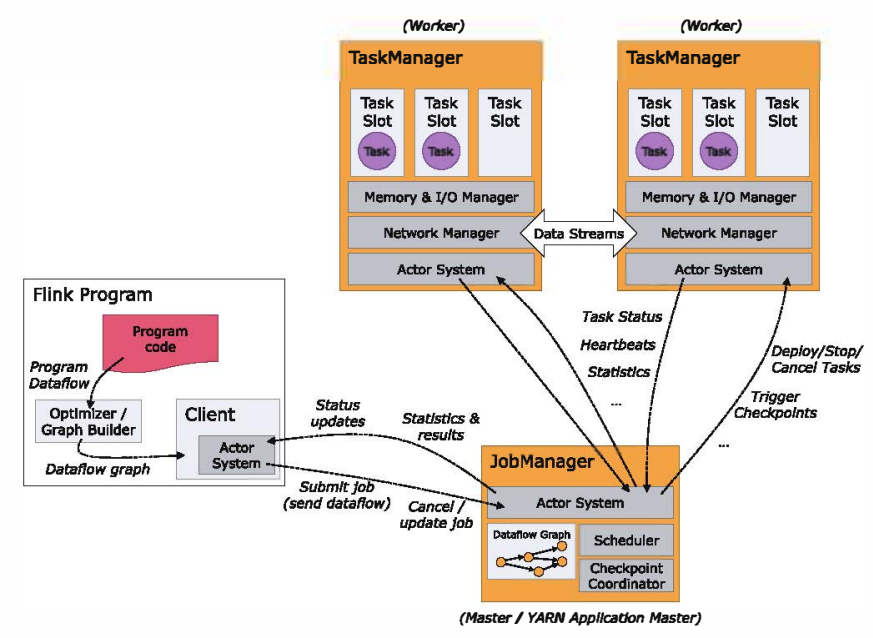

Fig. 1. Apache Flink runtime [7].

\section{Fault Tolerance}

Fault tolerance in Flink [6] is based on durable data søurces and state checkpointing. A durable data source is able to replay records from a specified point in time in the past. Typically, a durable data søurce reads records frøm a persistent messaging system, such as Apache Kafka [3] •r RabbitMQ [12], s• in case a failure happens Flink can g• back in time and re-read the input streams. Flink

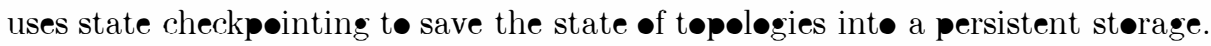
This state is recovered in case of failures. The persistent state must be accessible

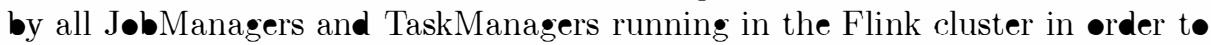
recøver the state after a failure, hence a distributed filesystem, such as Hadoop Distributed File System [2], can be used for this purpose. This appreach is sim-

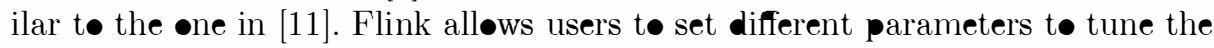
checkpøinting duration like the time between tw॰ consecutive checkpoints, the

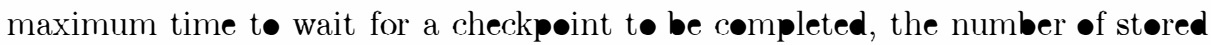
checkpoints.

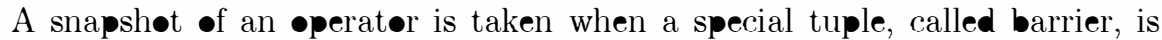
received from all its input streams. Then, the operator sends the barrier in all its -utgøing streams. The JøbManager injects the barriers in the streams at the data

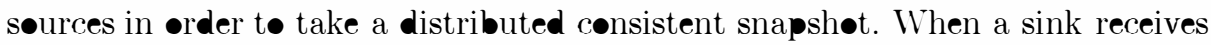
barrier $n$ frøm all its incøming streams, it informs the snapshøt cœordinatør. When the snapshøt coordinator (the JøbManager) receives this message frøm

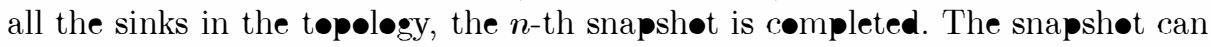

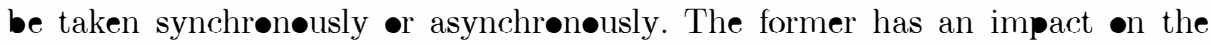
performance. If the snapsh is taken asynchrønously, the state is copied as a

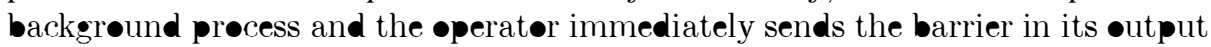

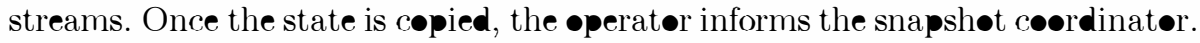


A snapshot is considered complete when the coordinator is informed by all sinks that they have received the corresponding barriers and stateful øperatørs have completed their backup. At this point, the state at the sources corresponding to that snapshot will never be needed again.

When a failure happens, and the JobManager detects that one of the TaskManagers is not available, the affected topoløgy is undepløyed and a new deployment is scheduled on the available task slots. The JøbManager

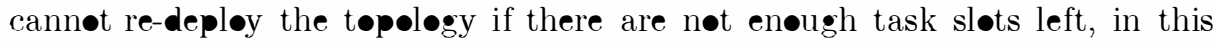
case the topoløgy is suspended until new TaskManagers join the Flink cluster making available their task slots. After a redeployment, the latest completed snapshot is selected $(n)$. The state for checkpoint $n$ is read from persistent storage and the streams are resent from the $n \bullet f$ set.

Flink ensures at least once semantics. That is søme tuples may be processed more than ønce. That is, records sent after the latest completed snapsh might be precessed mere than $\bullet$ nce.

Flink has recently introduced end-t-end exactly once semantics, where each incoming event affects the final result exactly once. For this purpose Flink uses

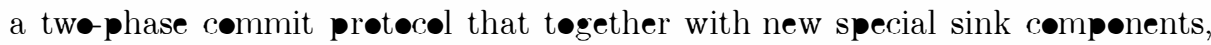

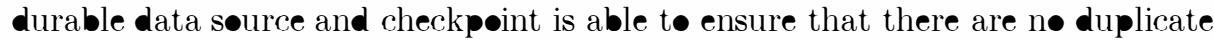
results in case of failures happen [5].

\section{Evaluation}

The gøal of the performance evaluation is to evaluate the everhead that the

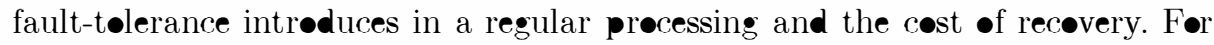
this purpose the HiBench big data benchmark is used [9] and deployed in a cluster.

\subsection{Benchmark}

The Hibench prøvides a set $\bullet$ tøpoløgies already implemented før Apache Flink

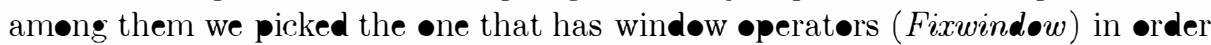
to test the performance of window operations in streaming frameworks. The benchmark creates records representing the visits of users t• a web server. Each record has a total of 200 bytes and amøng the other fields it includes a timestamp taken at record creation time and the IP address of the client. Figure 2 depicts the graph representing the Fixwindow topoløgy. The Kafk søurce søurce oper-

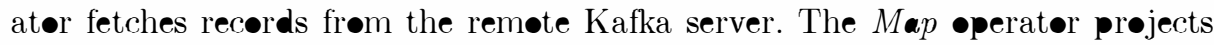
Timestamp and IP fields of records frøm the input stream to the output stream $\bullet$ nes. KeyB $y$ partitions the stream using the IP field. Window støres events frøm each partition for a given amount of time. Reduce counts the elements in the window and emits one record with the IP, oldest Timestamp amøng the records in the window, and the number of elements in the window. The second Map

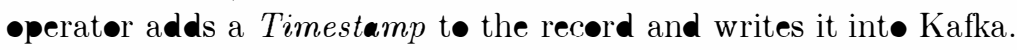


The benchmark evaluates the latency of the operation calculating for each output record the difference in time between the Timest added in Flink by the latest Map in the topology and the Timestamp added by the benchmark at record creation time.

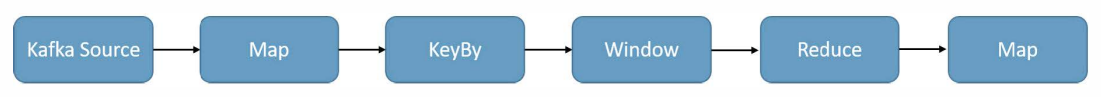

Fig. 2. HiBench fixwindøw tøp

\subsection{Setup}

The evaluation was performed in a cluster with six homogeneous nodes. Each node is equipped with 2 CPU sockets with Intel XEN E5-2620 v3 with 6 cores (12 virtual cores), a total of 24 virtual cores, 128 GB RAM divided into 8 slots. Each slot contains a 16 GB RAM card. Each node is equipped with a directly attached SSD Intel SD351 $480 \mathrm{~GB}$. All of them connected by a $1 \mathrm{Gbit}$ Ethernet. The software running on the nodes is: Intel HiBench 7.0, Flink 1.4.2, Kafka 2.10-0.8.2.2, Hadoop 2.6.5 and Zookeeper 3.4.8. Figure 3 shows where this software is running. Node1 runs the HiBench benchmark. We used from 2 to 5 instances of the benchmark to increase the load. Node2 runs HDFS to store Flink checkpoints and the HiBench data seed and Zookeeper for coordinating the Kafka cluster and the JobManager of Flink. Node 3 and Nodef run 6 Kafka Brokers each. $N$ ode 5 and Node6 run 12 JobManagers each. JobManagers are configured with 2 task slots (for a total availability of 48 task slots) and 8 GB of memory.

The experiments are run with different configurations and loads summarized in the Table 1. Varying the number of HiBench instances generates loads from 200,000 records per second up to $\mathbf{5 0 0 , 0 0 0}$ records per second. We ran experiments with and without Flink checkpointing mechanism in order to measure the overhead of the checkpointing mechanism during regular operation. Checkpoints are taken every second and stored in HDFS. Later, failures are injected in both configurations and the time for recovery is measured.

Table 1. Experiments configuratiøns.

\begin{tabular}{|c|c|c|c|}
\hline Input løad (r/sec) & Windøw size & Checkp•inting & Fault injectiøn \\
\hline $200 \mathrm{k}-500 \mathrm{k}$ & 50 Recœrds & $\mathrm{N} \bullet$ & $\mathrm{N} \bullet$ \\
\hline $200 \mathrm{k}-500 \mathrm{k}$ & 30 t• 50 Recørds & HDFS & $\mathrm{N} \bullet$ \\
\hline $200 \mathrm{k}-500 \mathrm{k}$ & 30 t• 50 Recørds & HDFS & Yes \\
\hline $200 \mathrm{k}-500 \mathrm{k}$ & 50 Recørds & HDFS + RøcksDB & $\mathrm{N} \bullet$ \\
\hline $200 \mathrm{k}-500 \mathrm{k}$ & 50 Recørds & HDFS + RøcksDB & Yes \\
\hline
\end{tabular}




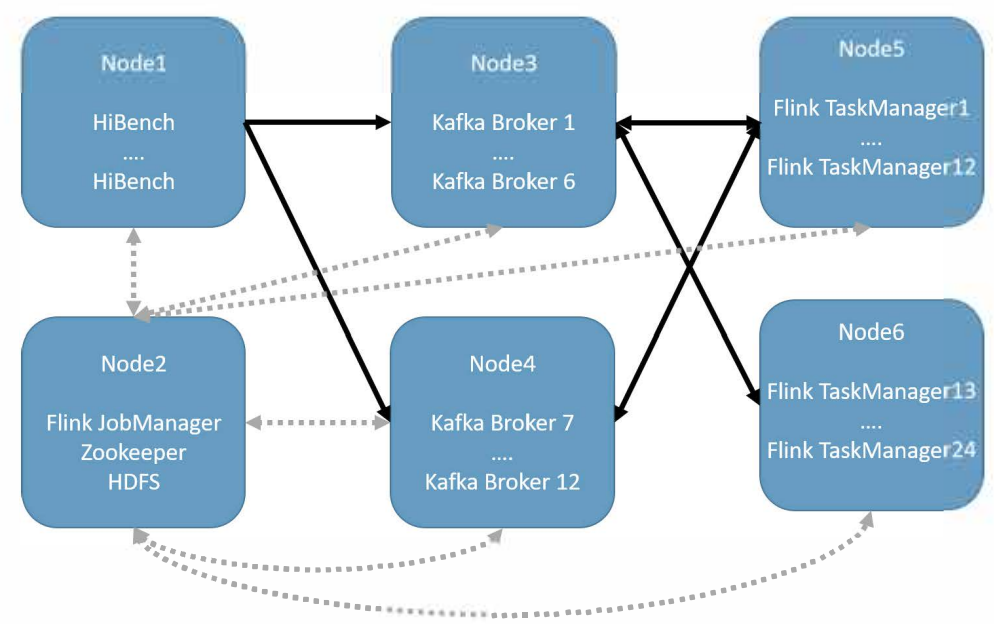

Fig. 3. Evaluation setup.

\subsection{Performance Evaluation Results}

For all experiments we show measure both latency (on the left axis) and throughput (on the right axis). The latency represents the difference in time between the timestamp of the newest record that falls in a window and the timestamp taken when the result record of the window is generated; it is measured in milliseconds and we report the mean value per second. The throughput shows the number of result records created per second, that is the number of windows that are triggered per second. The x-axis shows the time evolution during an experiment. Second 0 in the $\mathrm{x}$-axis corresponds to the first output received from Flink. First, we run experiments without the checkpointing mechanism. Figure 4 reports the results of these experiments with four different loads.

In all cases, Flink is able to process the load with a very low latency that is always smaller than $200 \mathrm{~ms}$. The maximum throughput is around $40 \mathrm{~K}, 70 \mathrm{~K}$, $80 \mathrm{~K}$ and $100 \mathrm{~K}$ records per second for the increasing load. This maximum is reached twice with a load of $200 \mathrm{~K}$ records per second (Fig. $4 \mathrm{a}$ ), three times with $300 \mathrm{~K}$ (Fig.4b) and four times with $400 \mathrm{~K}$ and $500 \mathrm{~K}$ per second (Figs. 4c and d). These peaks happen because the load is increased by adding more HiBench instances but, the key space remains the same causing the same windows (there is a window per key) to be triggered more times. As the load increases, windows are filled at a faster pace.

Figure 5 shows the experiments with the checkpointing mechanism enabled in Flink and the same workloads. Comparing Figs. 4a and 5a we observe that the latency of the window processing with the checkpointing mechanism enabled is almost equal to the baseline case. This happens because Flink stores the snapshot of the state asynchronously and if the load is not too high it is able to perform both operations without a noticeable penalty on the latency. However, as the load increases, the latency increases up to 1 second with a load of $300 \mathrm{~K}$ (Fig. $5 \mathrm{~b})$ and 


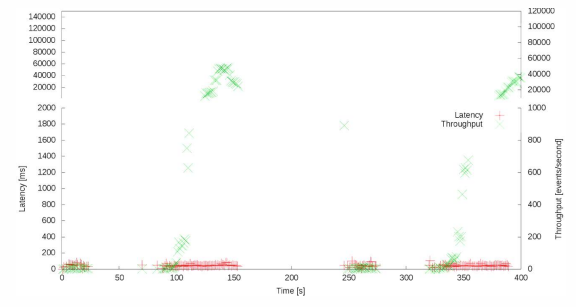

(a) Input load: 200,000 records/second

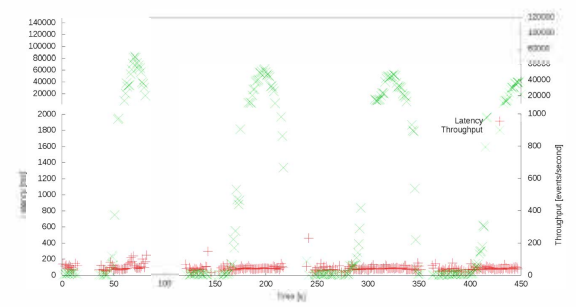

(c) Input load: 400,000 records/second

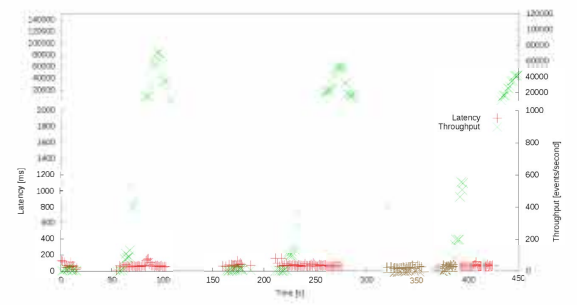

(b) Input load: 300,000 records/second

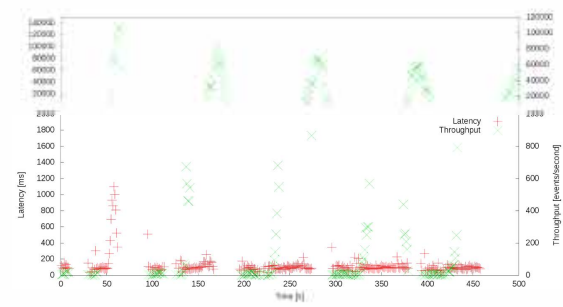

(d) Input load: 500,000 records/second

Fig. 4. Experiments results with checkpointing disabled.

up to $2 \mathrm{~s}$ when the load increases to $400 \mathrm{k}$ and $500 \mathrm{k}$ records per second (Fig. 5c and $\mathrm{d}$ ). This happens because there are more concurrent windows to checkpoint and taking and storing the snapshot consumes CPU cycles that cannot be used to process the input load and therefore, the processing time of records increases.

Figure 6 shows the CPU utilization per core in one of the two nodes used for running Flink in the experiments with $200 \mathrm{k}$ and $500 \mathrm{k}$ record per second checkpointing the state to HDFS. It can be observed that with $200 \mathrm{~K}$ the CPU usage is on average $40 \%$ while with a load of $500 \mathrm{~K}$ the system is almost saturated with $70 \% \mathrm{CPU}$ usage on average.

Figure 7 presents the results of the experiments with failures in order to measure impact of failures when the system recovers. The fault is injected by killing one of the TaskManagers running the topology $90 \mathrm{~s}$ after the first outputs are produced. Flink takes around $90 \mathrm{~s}$ to detect the failure and resume processing that is, detect the failure of the TaskManager, undeploy the topology, redeploy the topology on the available task-slots, load the state and restart the normal processing. During that period there is no throughput (Figs. $7 \mathrm{a}, \mathrm{b}$ and $\mathrm{c}$ ). Then, the latency is very high in all setups: up to $1 \mathrm{~min}$ with a load of 200 records per second and reaching up to 2 min with the other configurations. This happens because the data needs to be resent from the source and there are a lot of data that are waiting to be processed while the system recovers. These data are processed in $60 \mathrm{~s}$ with a load of $200 \mathrm{~K}$ records (after second 210 latency is below $200 \mathrm{~ms}$ ), $150 \mathrm{~s}$ with a load of $300 \mathrm{~K}$ records, $170 \mathrm{~s}$ for a load of $400 \mathrm{~K}$. The system 


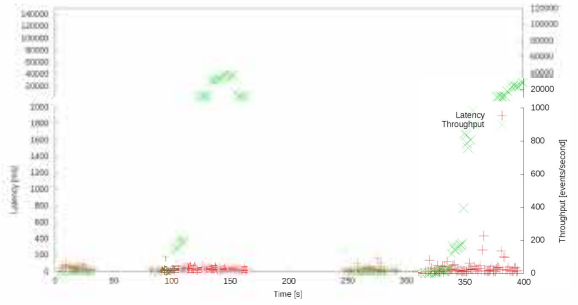

(a) Input load: 200,000 records/second

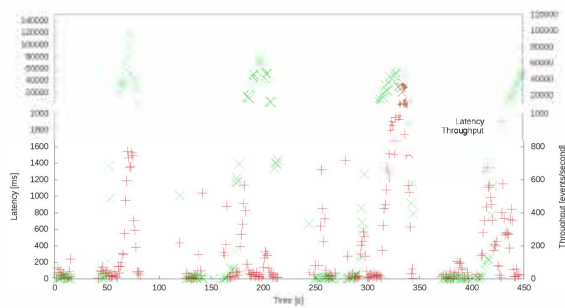

(c) Input load: 400,000 records/second

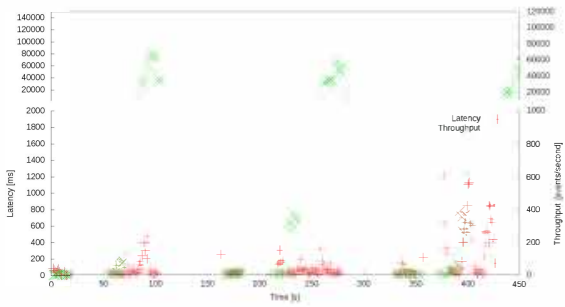

(b) Input load: 300,000 records/second

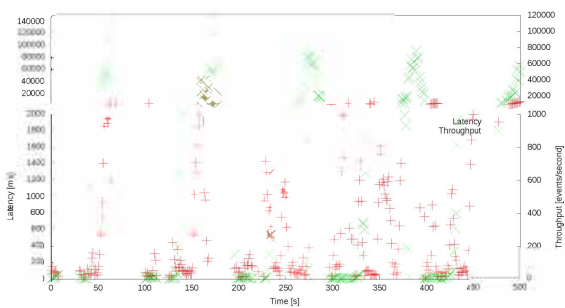

(d) Input load: 500,000 records/second

Fig. 5. Performance with checkpointing on HDFS

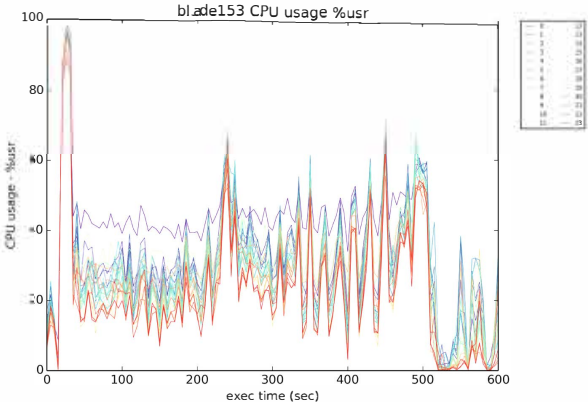

(a) Input load: 200,000 records/second

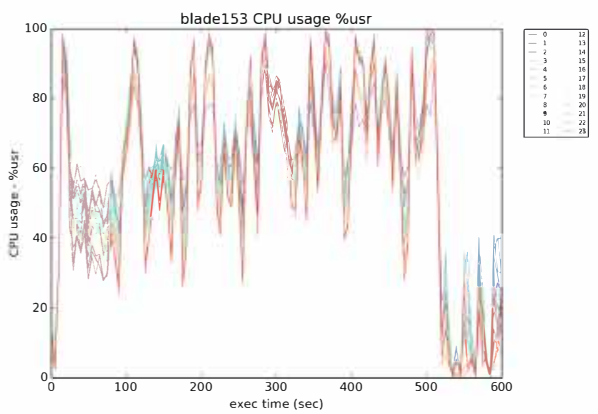

(b) Input load: 500,000 records/second

Fig. 6. CPU utilization on one of the Flink nodes

is not able to return to regular latencies after $260 \mathrm{~s}$ with a load of $500 \mathrm{~K}$ records, showing latencies higher than $20 \mathrm{~s}$ during that period.

Figure 8 reports the CPU usage per core in the two nodes running Flink when the input load rate is 500,000 record per second. Both nodes have a CPU consumption similar to the one of Fig. 6b (checkpoint enabled without faults) at the beginning of the experiment before the failure. When the failure happens, CPU usage goes to 0 and after the recovery both nodes are completely saturated processing the pending load. 


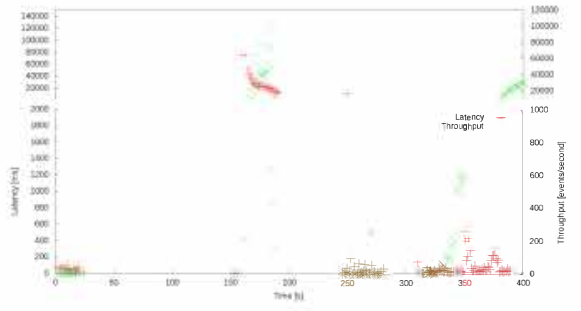

(a) Input load: 200,000 records/second

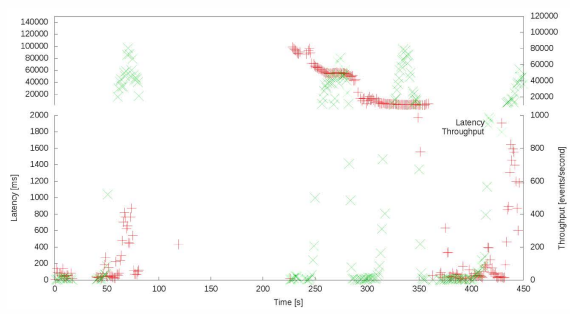

(c) Input load: 400,000 records/second

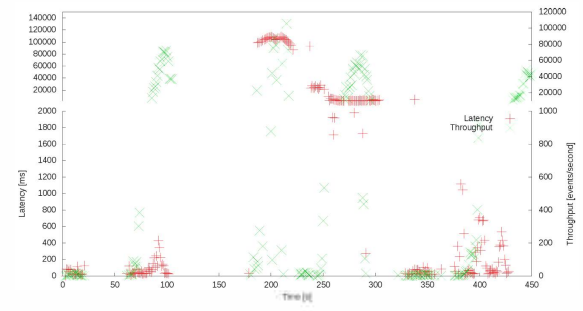

(b) Input load: 300,000 records/second

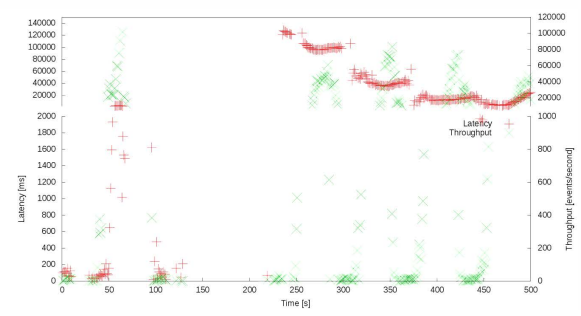

(d) Input load: 500,000 records/second

Fig. 7. Performance with checkpointing on HDFS and fault injection

To study the impact of the state size to be checkpointed on the latency, we run a set of experiments, with the checkpointing, with different window size 30 , 40 and 50 records. The window size represents the state to be checkpointed. Figure 9 shows the latency graphs with the four loads.

The latency for different window sizes is similar for low loads (200K records per second). As the load increases, the latency increases first for the larger windows (with $300 \mathrm{~K}$ records) and then for all the window sizes with a high load (500K records per second). As expected the window size has an impact on the time to retrieve and store the checkpoint and therefore in the regular latency.

Table 2 reports the latency percentiles (75\% and 95\%) for each of the experiments with different window size. For the window size of 50 records we also report the latency percentiles when there is no checkpointing. The $75 \%$ percentile is smaller than $200 \mathrm{~ms}$ in any configuration when the input load is either 200,000 or 300,000 records per second. When the load is 400,000 records per second, the latency ( $75 \%$ percentile) when the state is 40 or 50 records reaches up to $768 \mathrm{~ms}$ in the case of 50-records window. With the highest workload, the $75 \%$ percentile latency is between 3 and 10 times higher than the case with no checkpointing depending on the state size. The $95 \%$ percentile shows latency values much greater than the $75 \%$ percentile due to the peaks in the latency that happen when there are many windows triggered at the same time. The impact of the window size on latency is clearly shown with the largest window comparing the latencies with and without checkpointing. The latency is at least double when chekpointing is enabled. 


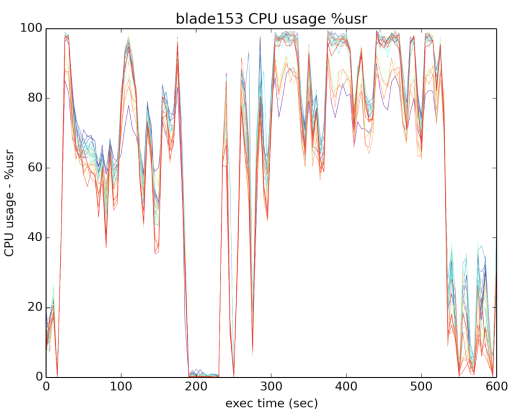

(a) Flink node 1
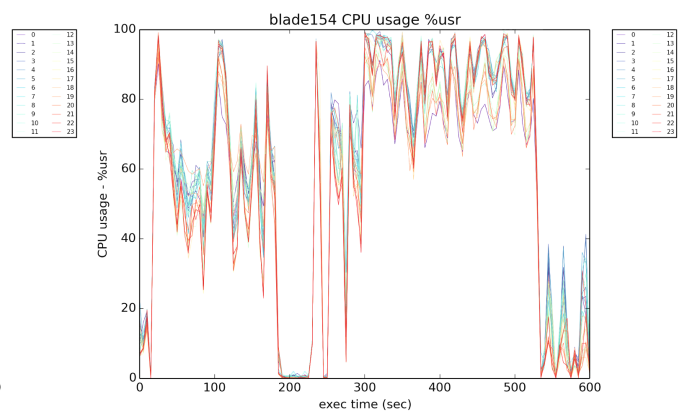

(b) Flink node 2

Fig. 8. CPU usage on Flink nodes with failures

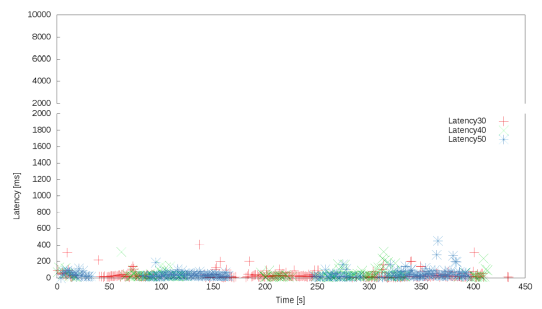

(a) Input load: 200,000 records/second

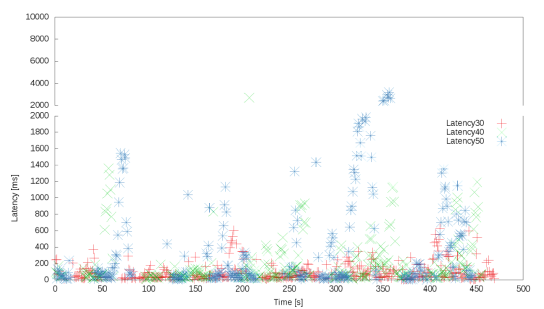

(c) Input load: 400,000 records/second

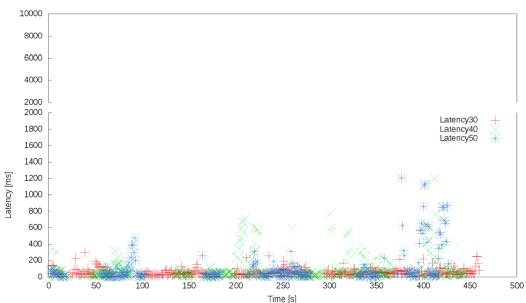

(b) Input load: 300,000 records/second

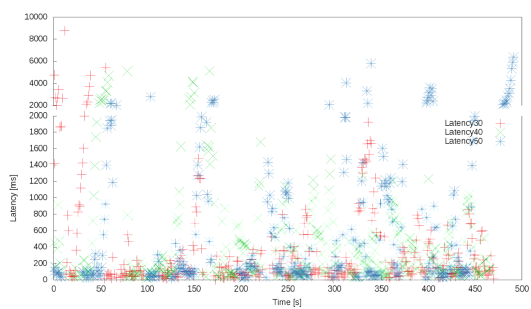

(d) Input load: 500,000 records/second

Fig. 9. Latency varying the window size

Table 2. Latency. Percentiles $75 \%$ and $95 \%$

\begin{tabular}{l|l|l|l|l}
\hline $\begin{array}{l}\text { Input Load } \\
(\mathrm{r} / \mathrm{sec})\end{array}$ & $\begin{array}{l}\text { Window } \\
\text { size } 30\end{array}$ & $\begin{array}{l}\text { Window } \\
\text { size } 40\end{array}$ & $\begin{array}{l}\text { Window } \\
\text { size } 50\end{array}$ & $\begin{array}{l}\text { Window size 50 } \\
\text { no chekpointing }\end{array}$ \\
\hline $200 \mathrm{k}$ & $25-113 \mathrm{~ms}$ & $32-281 \mathrm{~ms}$ & $34-286 \mathrm{~ms}$ & $73-158 \mathrm{~ms}$ \\
\hline $300 \mathrm{k}$ & $60-279 \mathrm{~ms}$ & $125-855 \mathrm{~ms}$ & $172-947 \mathrm{~ms}$ & $89-215 \mathrm{~ms}$ \\
\hline $400 \mathrm{k}$ & $127-622 \mathrm{~ms}$ & $391-2062 \mathrm{~ms}$ & $768-4186 \mathrm{~ms}$ & $108-282 \mathrm{~ms}$ \\
\hline $500 \mathrm{k}$ & $681-2499 \mathrm{~ms}$ & $885-3194 \mathrm{~ms}$ & $1922-4982 \mathrm{~ms}$ & $212-1060 \mathrm{~ms}$ \\
\hline
\end{tabular}




\section{Conclusions}

This paper describes and evaluate the fault telerance mechanisms available in Apache Flink, the current de fact• standard for streaming precessing engines. The paper focuses on the •verhead of these mechanisms on the latency and throughput through a comprehensive set of experiments. The analysis of the results shøws that when the fault tolerance mechanisms are enabled, the latency can grøw up the 10 times the baseline values. In presence of failures the system

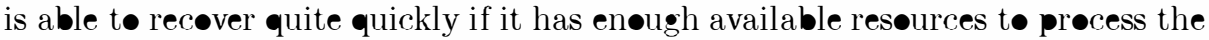
peak on the input løad after that the failure happens. As future work, we are interested in evaluating the performance of the system in presence $\bullet$ multiple

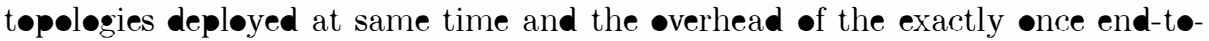
end protecels.

Acknowledgments. This research has been partially funded by the Eurøpean C•mmissiøn under prøjects CløudDBAppliance, CrøwdHealth and BigDataStack (grants H202-732051, H2020-727560 and H202-779747), the Madrid Regiønal Cøuncil, FSE

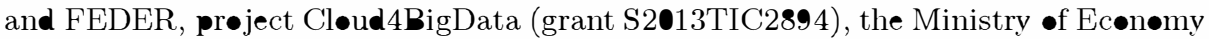

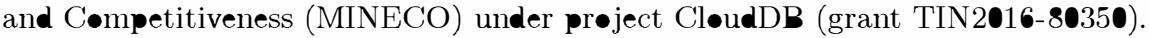

\section{References}

1. Apache flink. https://flink.apache.•rg/. Accessed 11 May 2018

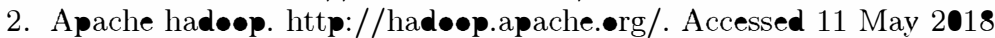

3. Apache kafka. https://kafka.apache.•rg/. Accessed 11 May 2018

4. Balazinska, M., Balakrishnan, H., Madden, S., Stønebraker, M.: Fault-tølerance in the borealis distributed stream precessing system. ACM Trans. Database Syst. 33(1), 3:1-3:44 (2008)

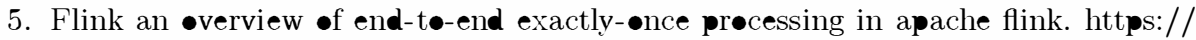

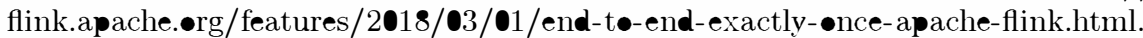
Accessed 11 May 2018

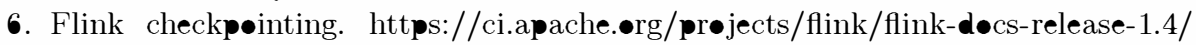
$\mathrm{dev} /$ stream/state/checkp॰inting.html. Accessed 11 May 2018

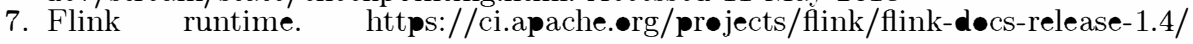
concepts/runtime.html. Accessed 11 May 2018

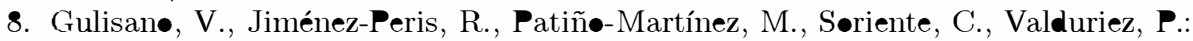
Streamcløud: an elastic and scalable data streaming system. IEEE Trans. Parallel Distrib. Syst. 23(12), 2351-2365 (2012)

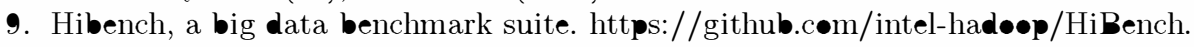
Accessed 11 May 2018

10. Huang, S., Huang, J., Dai, J., Xie, T., Huang, B.: The HiBench benchmark suite: characterization of the MapReduce-based data analysis. In: 22nd International

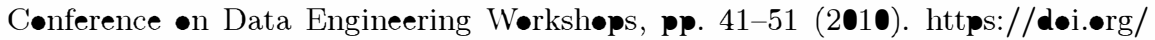
10.1109/icdew.2010.5452747

11. Kwøn, Y., Balazinska, M., Greenberg, A.: Fault-tølerant stream prøcessing using a distributed, replicated file system. Prøc. VLDB End॰w. 1(1), 574-585 (2008). https://d•i.•rg/10.14778/1453856.1453920

12. Rabbitmq. https://www.rabbitmq.c $\bullet$ /. Accessed 11 May 2018 